\title{
Magnetotransport in dilute 2D Si-MOSFET system
}

\author{
M. V. Cheremisin \\ A.F.Ioffe Physical-Technical Institute, St.Petersburg, Russia
}

(Dated: November 19, 2018)

\begin{abstract}
The beating pattern of Shubnikov-de Haas oscillations is reproduced in both the crossed and tilted magnetic field configuration and in presence of zero-field valley splitting in Si-MOSFET system. The consequences of IQHE in extremely dilute 2DEG are discussed.

PACS numbers: $71.30+\mathrm{h}, 71.27 .+\mathrm{a}, 73.40 . \mathrm{Qv}$
\end{abstract}

A great deal of interest has been focussed on the anomalous transport behavior [1] of a wide variety of lowdensity $2 \mathrm{D}$ systems. It has been found that, below some critical density, the cooling causes an increase in resistivity, whereas in the opposite high density case the resistivity decreases. Another unusual property of low-density $2 \mathrm{D}$ systems is their response to perpendicular magnetic field. In dilute Si-MOSFET system the spin susceptibility known to be strongly enhanced, therefore results in magnetotransport features associated mostly with spin. Although numerous theories have been put forward to account for these effects, the origin of the above behavior is still the subject of a heated debate. In present paper we investigate the beating pattern of Shubnikov-de Haas $(\mathrm{SdH})$ oscillations caused by zero-field valley splitting in Si-MOSFET system. Then, we analyze the $\mathrm{SdH}$ beating pattern for the crossed magnetic field configuration case. Magnetotransport in extremely dilute 2DEG subjected into quantizing magnetic fields is discussed.

In contrast to conventional $\mathrm{SdH}$ formalism extensively used to reproduce low-field data we allude to alternative approach [2] seems to give an overwhelming efforts to resolve magnetotransport problem within both $\mathrm{SdH}$ and IQHE regimes. Based on a thermodynamic approach, in Ref. 2] has been calculated the magnetoresistivity of a $2 \mathrm{D}$ electron gas, assumed nevertheless dissipationless in a strong quantum limit. Standard measurements, with extra current leads, define the magnetoresistivity caused by a combination of Peltier and Seebeck effects. 3], 4] The current causes heating(cooling) at the first(second) sample contacts, due to the Peltier effect. The contact temperatures are different. The measured voltage is equal to the Peltier effect-induced thermoemf which is linear in current. As a result, the magnetoresistivity is nonzero as $I \rightarrow 0$. The resistivity found to be a universal function of magnetic field and temperature, expressed in fundamental units $h / e^{2}$.

The Si-MOSFET energy spectrum modified with respect to valley and spin splitting yields

$$
\varepsilon_{n}=\hbar \omega_{c}(n+1 / 2) \pm \frac{\Delta_{s}}{2} \pm \frac{\Delta_{v}}{2}
$$

where $n=0,1$. is the LL number, $\omega_{c}=\frac{e B_{\perp}}{m c}$ the cyclotron frequency, $\Delta_{s}=g^{*} \mu_{B} B$ the Zeeman splitting, $g^{*}$ the effective $g$-factor, $B=\sqrt{B_{\perp}^{2}+B_{\|}^{2}}$ the total magnetic field. Then, $\Delta_{v}[\mathrm{~K}]=\Delta_{v}^{0}+0.6 B_{\perp}[\mathrm{T}]$ is the den- sity independent [5] valley splitting. In contrast to valley splitting, the spin susceptibility $\chi=\frac{g^{*} m}{2 m_{0}}$ ( here, $m_{0}$ is the free electron mass ) known to exhibit strong enhancement upon 2D carrier depletion. The latter result is confirmed independently by magnetotransport measurements in tilted magnetic field [6], 7], perpendicular field [8] and beating pattern of $\mathrm{SdH}$ oscillations [9] in crossed fields.

Recall that in strong magnetic fields $\hbar \omega_{c} \gg k T, \hbar / \tau$ the electrons can be considered dissipationless, therefore $\sigma_{x x}, \rho_{x x} \simeq 0$. Here, $\tau$ is the momentum relaxation time. Under current carrying conditions the only reason for finite longitudinal resistivity seems to be thermal correction mechanism discussed in Ref.[3]. Following Ref. 2] one obtains

$$
\rho=\rho_{y x} \frac{\alpha^{2}}{L}
$$

where $\alpha$ is the thermopower, $\rho_{y x}^{-1}=N e c / B_{\perp}$ the Hall resistivity, $N=-\left(\frac{\partial \Omega}{\partial \mu}\right)_{T}$ the $2 \mathrm{D}$ density, $\Omega=$ $-k T \Gamma \sum_{n} \ln \left(1+\exp \left(\frac{\mu-\varepsilon_{n}}{k T}\right)\right)$ the thermodynamic potential modified with respect to abovementioned energy spectrum, $\Gamma=\frac{e B_{\perp}}{h c}$ the zero-width LL DOS. In actual fact, in strong magnetic fields $2 \mathrm{D}$ thermopower is a universal quantity 10], proportional to the entropy per electron: $\alpha=-\frac{S}{e N}$, where $S=-\left(\frac{\partial \Omega}{\partial T}\right)_{\mu}$ is the entropy. Both $S, N$, and, thus $\alpha, \rho$ are universal functions of $\xi$ and the dimensionless magnetic field $\hbar \omega_{c} / \mu=4 / \nu$, where $\nu=N_{0} / \Gamma$ is the conventional filling factor, $N_{0}=\frac{2 m \mu}{\pi \hbar^{2}}$ is the zero-field density of strongly degenerate $2 \mathrm{DEG}$.

Using Lifshitz-Kosevich formalism, asymptotic formulae can be easily derived for $N, S$, and hence for $\rho_{y x}, \rho$, valid within low temperature, magnetic field limit $\nu^{-1}, \xi<1$ :

$$
\begin{aligned}
& N=N_{0} \xi F_{0}(1 / \xi)+2 \pi \xi N_{0} \sum_{l=1}^{\infty} \frac{(-1)^{l} \sin \left(\frac{\pi l \nu}{2}\right)}{\sinh \left(r_{l}\right)} R(\nu), \\
& S=S_{0}-2 \pi^{2} \xi k N_{0} \sum_{l=1}^{\infty}(-1)^{l} \Phi\left(r_{l}\right) \cos \left(\frac{\pi l \nu}{2}\right) R(\nu),
\end{aligned}
$$

where $S_{0}=k N_{0} \frac{d}{d \xi}\left[\xi^{2} F_{1}(1 / \xi)\right]$ is the entropy at $B_{\perp}=$ $0, F_{n}(z)$ the Fermi integral, $\Phi(z)=\frac{1-z \operatorname{coth}(z)}{z \cdot \sinh (z)}, r_{l}=$ $\pi^{2} \xi \nu l / 2$ the dimensionless parameter. Then, $R(\nu)=$ 


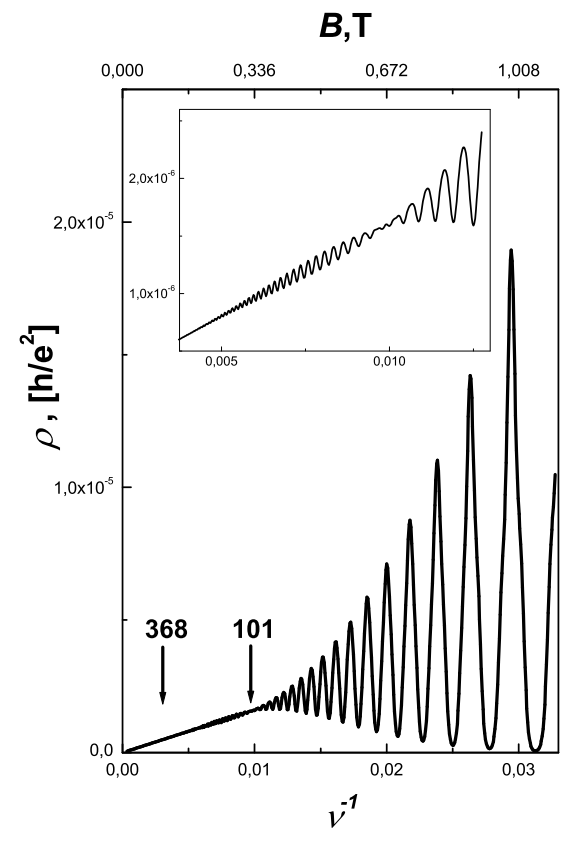

FIG. 1: $\mathrm{SdH}$ oscillations at $T=0.3 \mathrm{~K}$ for Si-MOSFET sample[11]: $N_{0}=8.39 * 10^{11} \mathrm{~cm}^{-2}$, spin susceptibility $\chi=$ 0.305 and valley splitting $\Delta_{v}[\mathrm{~K}]=\Delta_{v}^{0}+0.6 B_{\perp}[\mathrm{T}]$. Zero-field valley splitting $\Delta_{v}^{0}=0.92 K$ is a fitting parameter. Arrows depict the beating nodes at $i=1,3$. Inset: the enlarge plot of the beating node from the main panel.

$\cos (\pi l s) \cos (\pi l v)$ is the form-factor, $s=\frac{\Delta_{s}}{\hbar \omega_{c}}=\chi \frac{B}{B_{\perp}}$ the dimensionless Zeeman spin splitting, $v=\frac{\Delta_{v}}{\hbar \omega_{c}}=$ $\frac{\Delta_{v}^{0} \nu}{4 \mu}+0.12$ the dimensionless valley splitting.

Let us first consider zero- $B_{\|}$case, when the Zeeman spin splitting is reduced to field-independent constant, i.e. $s=\chi$. Then, in low- $T, B_{\perp}$ limit the valley splitting $\Delta_{v}^{0}$ known to be resolved 11], therefore leads to beating of SdH oscillations. For actual first-harmonic case( i.e. $l=1$ ), the beating nodes can be observed when $\cos (\pi v)=0$, or $\nu_{i}^{v}=\frac{4 \mu(i / 2-0.12)}{\Delta_{v}^{0}}$, where $i=1,3$. is the beating node index. For 2DEG parameters( see Fig 1) reported in Ref. [1] we estimate $\nu_{1}^{v}=101$, therefore $\Delta_{v}^{0}=0.92 \mathrm{~K}$. The second node is expected to appear at $\nu_{2}^{v}=368$. However, $\mathrm{SdH}$ oscillations are, in fact, resolved when $\nu \leq 1 / \xi=203$, therefore the second beating node was not observed in experiment [11]. Moreover, the observed disappearance of the first beating node upon 2D carrier depletion $N<3 * 10^{11} \mathrm{~cm}^{-2}$ is governed by the same condition because in this case $\nu \leq 1 / \xi=73$ being of the order of the first beating node. Note that suppression the beating nodes at higher densities $\left(N>9 * 10^{11} \mathrm{~cm}^{-2}\right)$ reported in Ref. 11] is, however, unexpected within our simple scenario.

We now analyze the case of low-density 2D system in strong magnetic field with the only lowest LLs occupied. For extremely dilute $2 \operatorname{DEG}\left(N \simeq 10^{11} \mathrm{~cm}^{-2}\right)$ the energy

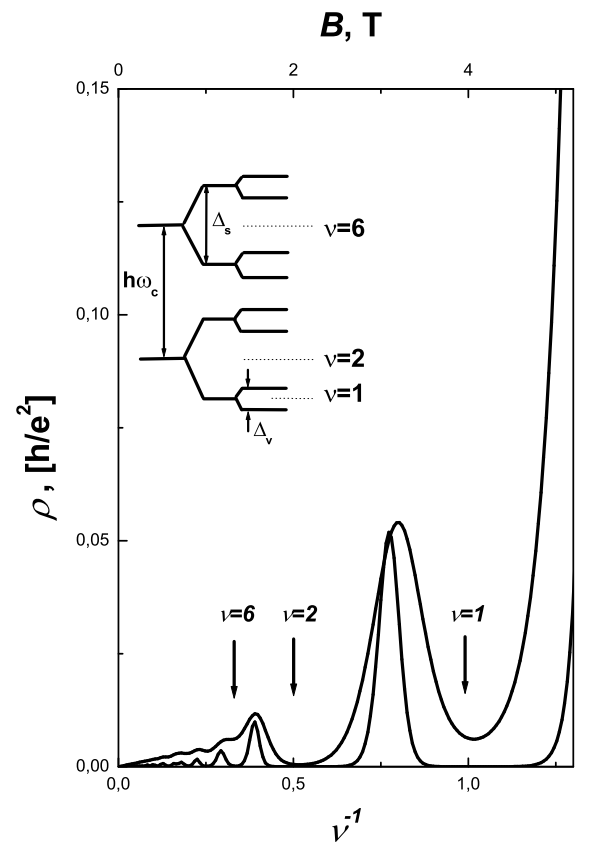

FIG. 2: Magnetoresistivity at $T=0.36$; $0.18 \mathrm{~K}$ for dilute 2DEG Si-MOSFET [12]: $N_{0}=10^{11} \mathrm{~cm}^{-2}$, spin susceptibility $\chi=0.5$ and valley splitting denoted in Fig 1 Inset: Energy spectrum specified by Eq1] for two lowest LLs

spectrum( Fig 3 inset) known to be strongly affected by enhanced spin susceptibility. In contrast to high density case with cyclotron minima occur at $\nu=4,8,12$.., in dilute 2DEG the only spin minima $(\nu=2,6,10 .$. are observed. 12] As expected, the spin (cyclotron) minima fillings are proportional to the odd( even ) numbers multiplied by factor of two due to the valley degeneracy. In stronger fields magnetoresistivity data exhibit $\nu=1$ minimum associated with valley splitting. With the help of energy spectrum implied by Eq.(11) one can easily find that the last minima occur when the Fermi level lies between the lowest valley-split LLs, i.e. $\mu=\hbar \omega_{c}(1-\chi) / 2$. The sequence of minima at $B=4,2,0.66 \mathrm{~T}$ reported in Ref. 12 provides the independent test for spin susceptibility in high- $B_{\perp}$ limit. In Fig 2 we represent the magnetoresistivity specified by $\mathrm{Eq} 2$ and then use $\chi=0.5$ in order to fit the observed minima sequence. Surprisingly, the value of spin susceptibility is lower than that $\chi=0.86$ extracted from crossed-field $\mathrm{SdH}$ beating pattern analysis [9]. We attribute the above discrepancy to, for example, the possible magnetic field dependence of spin susceptibility.

Finally, we focus on magnetotransport problem in crossed magnetic field configuration. Following experiments 11] we further neglect the zero-field valley splitting for actual high density case $\left(N>9 * 10^{11} \mathrm{~cm}^{-2}\right)$. At fixed parallel magnetic field the dimensionless Zeeman splitting yields $s=\chi \sqrt{1+\nu^{2} / \nu_{\|}^{2}}$, where we intro- 


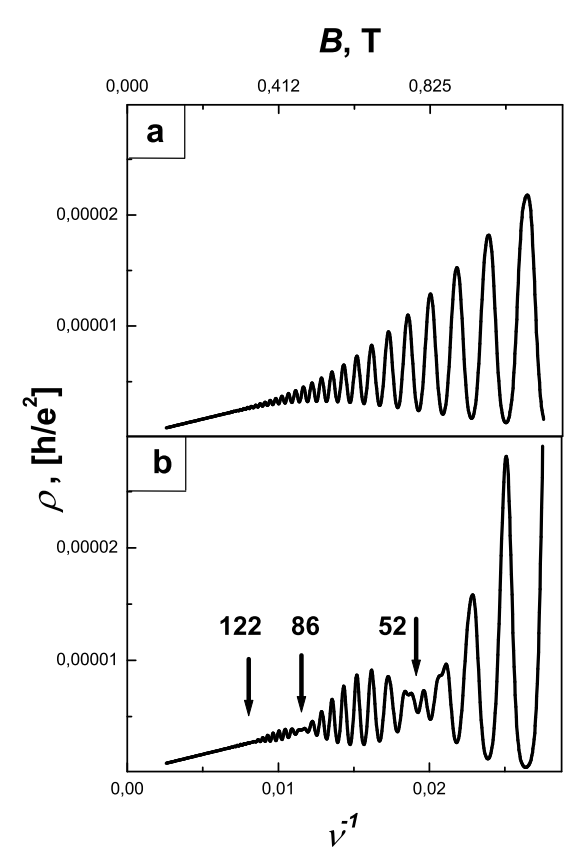

FIG. 3: SdH beating pattern oscillations at $T=0.35 \mathrm{~K}$ for Si-MOSFET sample [9]: $\quad N_{0}=10.6 * 10^{11} \mathrm{~cm}^{-2}$, spin susceptibility $\chi=0.27, \Delta_{v}^{0}=0$ and a) $\left.B_{\|}=0 \mathrm{~b}\right) B_{\|}=4.5 \mathrm{~T}$ $\left(\nu_{\|}=9.25\right)$. Arrows depict the beating nodes at $j=3,5,7$

duce an auxiliary "filling factor" $\nu_{\|}=\frac{h c N_{0}}{e B_{\|}}$associated with the parallel field. Within low- $B_{\perp}$ limit the parallel field induced spin splitting result in the beating of $\mathrm{SdH}$ oscillations as well. One can easily derive the condition for $\mathrm{SdH}$ beating nodes as follows $\cos (\pi s)=0$ or $\nu_{j}^{s}=\nu_{\|} \sqrt{(j / 2 \chi)^{2}-1}$, where $j=1,3 \ldots$. The sequence of the beating nodes observed in Ref. [9] allowed the authors to deduce the density dependence of the spin susceptibility. As an example, for 2DEG parameters [9] in Fig [3 we reproduce the magnetoresistivity implied by Eqs.(2), (3). The phase of $\mathrm{SdH}$ oscillations remains the same between the adjacent beating nodes, and changes by $\pi$ through the node in consistent with experiments.

We now consider 2DEG magnetotransport in tilted configuration with the sample rotated in a constant magnetic field [6, 7, 13]. In this case, the $\mathrm{SdH}$ beating pattern known to depend on the spin polarization degree $p=\frac{\Delta_{s}}{2 \mu}=\frac{2 \chi}{\nu_{t o t}}$, where we introduce the auxiliary "filling factor" $\nu_{t o t}=\frac{h c N_{0}}{e B}$, associated with the total magnetic field. Conventionally, the spin polarization degree is related to parallel field $B_{c}$ required for complete spin polarization, therefore $p=\frac{B}{B_{c}}$. Performing a minor modification in Eq2 namely that $s=\chi \frac{\nu}{\nu_{t o t}}$, in Fig 4 we represent the magnetoresistivity as a function of filling factor for 2DEG plane rotated with respect to constant magnetic field $\mathrm{B}=18 \mathrm{~T}$ (see Ref. 13$]$ ). For simplicity, we omit zero-field valley splitting. Then, arguing the LLs spreading is neglected within our simple ap-
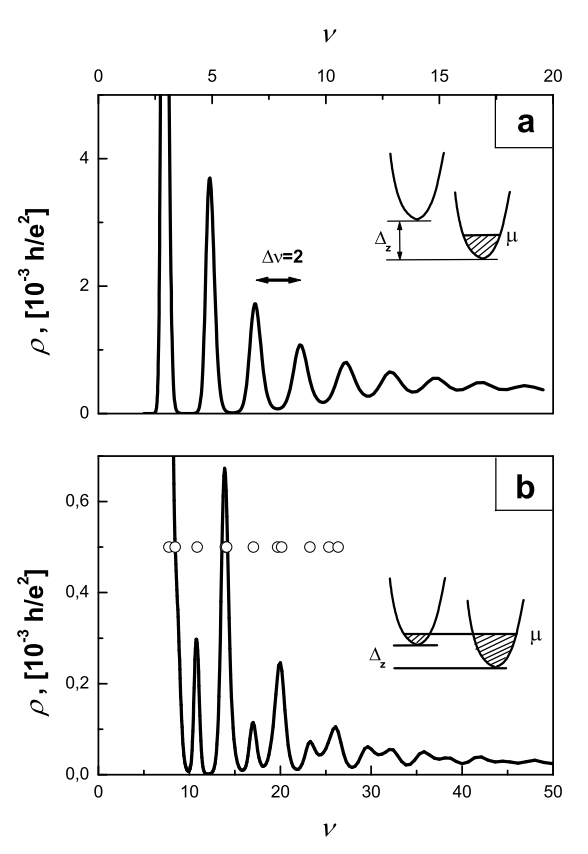

FIG. 4: Small-angle SdH oscillations at $T=1.35 \mathrm{~K}$ for SiMOSFET system [13]: a) spin polarized electrons $(p=1.01)$ at $N_{0}=3.72 * 10^{11} \mathrm{~cm}^{-2}$, spin susceptibility $\chi=0.42[9]$, "effective filling factor" $\nu_{t o t}=0.83$ and b)partially polarized case $(p=0.29)$ at $N_{0}=9.28 * 10^{11} \mathrm{~cm}^{-2}$, spin susceptibility $\chi=0.30[9]$ and $\nu_{t o t}=2.06$. Maxima positions are represented by open dots. Insets: schematic band diagrams at $B=B_{\|}$

proach, we use somewhat higher temperature compared to that in experiment 13. For spin polarized system $\mathrm{SdH}$ oscillations $(p=1.01 \mathrm{Fig}$ (4) $)$ is caused by the only lowest valley-degenerated spin-up subband. At low temperatures, the valley-splitting associated deep at $\nu=3$ found to be resolved. With the help of energy spectrum, specified by Eq⿴ the high-filling maxima occur at $\frac{4(N+1 / 2)}{1+p} \sim$ $2 N+1$, therefore have a period $\Delta \nu=2$. In contrast, the partially polarized high-density 2 DEG case $(p=0.29)$ depicted in Fig $4 \mathrm{~b}$ demonstrates rather complicated beating pattern caused by the both spin-up and spin-down subbands. One can easily demonstrate that high-filling maxima occur at $\frac{4(N+1 / 2)}{1 \pm p}$ (dots in Fig 近 b), thus depend on spin polarization degree. The ratio of oscillation frequencies of two spin subbands is $f^{\downarrow} / f^{\uparrow}=\frac{1-p}{1+p}$ being consistent with experiment [13]. At a moment, we, however, cannot explain the puzzling behavior of low-filling magnetoresistivity known(Ref. [7], 12]) to be insensitive to parallel field component.

We emphasize that the data represented in Fig 14 differs with respect to those provided by conventional formalism in the following aspects: i) low-field $\left(\omega_{c} \tau \leq 1\right)$ quantum interference and classical negative magnetoresistivity background is excluded within our approach and ii) in contrast to conventional $\mathrm{SdH}$ analysis, our approach 
determines (at $\left.\omega_{c} \tau \gg 1\right)$ the absolute value of magnetoresistivity, and, moreover provides the continuous transition SdH-to-QHE regime $\left(\hbar \omega_{c} \gg k T\right)$. Minor point is that our approach predicts somewhat lower SdH oscillations amplitude compared to that in experiment. However, in IQHE regime the magnitude of magnetoresistivity is well comparable( see Ref.[2]) with experimental values.
In conclusion, we demonstrate the relevance of the approach suggested in Ref. 2]) regarding to low-field beating pattern SdH oscillations in both crossed and tilted magnetic field configuration. Then, we examine the features concerning IQHE in dilute Si-MOSFET system.

This work was supported by RFBR(grant 03-0217588), and LSF(HPRI-CT-2001-00114, Weizmann Institute)
[1] For review, see E.Abrahams, S.V. Kravchenko, M.P.Sarachik: Rev. Mod. Phys. 73251 (2001).

[2] M.V. Cheremisin, Proceedings on NATO ASI, Windsor, UK, (2001), cond-mat/0102153

[3] C.G.M. Kirby and M.J. Laubitz, Metrologia 9, 103 (1973).

[4] M.V. Cheremisin, Sov.Phys.JETP 92, 357 (2001).

[5] V.M. Pudalov etal, Sov.Phys.JETP 62, 1084, (1985).

[6] F.F.Fang and P.J.Stiles, Phys.Rev. 174, 823 (1968).
[7] T. Okamoto etal, Phys. Rev. Lett. 82, 3875 (1999).

[8] S.V. Kravchenko etal, Solid State Commun. 116, 495 (2000).

[9] V.M. Pudalov etal, Phys. Rev. Lett. 88, 196404 (2002).

[10] S.M.Girvin and M.Jonson, J.Phys.C, 15, L1147 (1982).

[11] V.M. Pudalov etal, cond-mat/0104347

[12] S.V. Kravchenko etal, Phys.Rev.B 58, 3553 (1998).

[13] S.A. Vitkalov etal, Phys.Rev.B 64, 073101 (2001). 